\title{
ON THE RIGIDITY THEOREMS FOR LAGRANGIAN TRANSLATING SOLITONS IN PSEUDO-EUCLIDEAN SPACE II
}

\author{
RONGLI HUANG AND RUIWEI XU
}

\begin{abstract}
Let $u$ be a smooth convex function in $\mathbb{R}^{n}$ and the graph $M_{\nabla u}$ of $\nabla u$ be a space-like translating soliton in pseudo-Euclidean space $\mathbb{R}_{n}^{2 n}$ with a translating vector $\frac{1}{n}\left(a_{1}, a_{2}, \cdots, a_{n} ; b_{1}, b_{2}, \cdots, b_{n}\right)$, then the function $u$ satisfies

$$
\operatorname{det} D^{2} u=\exp \left\{\sum_{i=1}^{n}-a_{i} \frac{\partial u}{\partial x_{i}}+\sum_{i=1}^{n} b_{i} x_{i}+c\right\} \quad \text { on } \quad \mathbb{R}^{n}
$$

where $a_{i}, b_{i}$ and $c$ are constants. The Bernstein type results are obtained in the course of the arguments.
\end{abstract}

\section{INTRODUCTION}

Consider the logarithmic Monge-Ampère flow, (cf.[17])

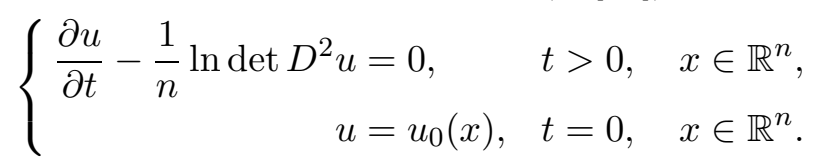

By Proposition 2.1 in [19], there exists a family of diffeomorphisms

$$
r_{t}: \mathbb{R}^{n} \rightarrow \mathbb{R}^{n}
$$

such that the map

$$
\begin{aligned}
F(x, t) & =\left(r_{t}(x), D u\left(r_{t}(x), t\right)\right) \subset \mathbb{R}_{n}^{2 n}, \\
F_{0}(x) & =\left(x, D u_{0}(x)\right)
\end{aligned}
$$

satisfies the mean curvature flow in pseudo-Euclidean space:

$$
\left\{\begin{aligned}
\frac{d F}{d t} & =\vec{H}, \\
F(x, 0) & =F_{0}(x),
\end{aligned}\right.
$$

where $\vec{H}$ is the mean curvature vector of the sub-manifold defined by $F$.

Definition 1.1. Assume that $u_{0}(x) \in C^{2}\left(\mathbb{R}^{n}\right)$. We call $u_{0}(x)$ satisfying condition (S), if

$$
\Lambda I \geq D^{2} u_{0}(x) \geq \lambda I, \quad x \in \mathbb{R}^{n} .
$$

Here $\Lambda, \lambda$ are two positive constants and $I$ is the identity matrix.

2010 Mathematics Subject Classification. Primary 53A10; Secondary 53C44.

Keywords and phrases. logarithmic Monge-Ampère flow, space-like translating soliton, Legendre transformation. 
The first author established the long time existence result of the logarithmic Monge-Ampère flow [19].

Proposition 1.1. Let $u_{0}: \mathbb{R}^{n} \rightarrow \mathbb{R}$ be a $C^{2}$ function which satisfies condition (S). Then there exists a unique strictly convex solution of (1.1) such that

$$
u(x, t) \in C^{\infty}\left(\mathbb{R}^{n} \times(0,+\infty)\right) \cap C\left(\mathbb{R}^{n} \times[0,+\infty)\right)
$$

where $u(\cdot, t)$ satisfies condition (S). More generally, for $l \in\{3,4,5 \cdots\}$ and $\varepsilon_{0}>0$, there holds

$$
\sup _{x \in \mathbb{R}^{n}}\left|D^{l} u(x, t)\right|^{2} \leq C, \quad \forall t \in\left(\varepsilon_{0},+\infty\right),
$$

where $C$ depends only on $n, \lambda, \Lambda, \frac{1}{\varepsilon_{0}}$.

More generally, the following decay estimates were derived in [20].

Proposition 1.2. Assume that $u(x, t)$ is a strictly convex solution of (1.1), and $u(\cdot, t)$ satisfies condition (S). Then there exists a positive constant $C$ depending only on $n, \lambda, \Lambda, \frac{1}{\varepsilon_{0}}$, such that for all $l \in\{3,4,5 \cdots\}$ there holds

$$
\sup _{x \in \mathbb{R}^{n}}\left|D^{l} u(x, t)\right|^{2} \leq \frac{C}{t^{l-2}}, \quad \forall t \geq \varepsilon_{0} .
$$

Self-shrinking solutions of the mean curvature flow are determined by the following quasi-linear elliptic systems

$$
\vec{H}=-\frac{X^{N}}{2}
$$

In the ambient Euclidean space, the self-shrinkers has been considered in [1], [16], [6], [7], 14], 13]. In the ambient pseudo-Euclidean space, the self-shrinking graphs with high codimensions can be seen in [1, [20, [10, 9]. The solutions are hoped to give a better understanding of the flow at type I singularities by Huiskens monotonicity formula. Let $M=\left\{(x, D u(x)) \mid x \in \mathbb{R}^{n}\right\}$ be a space-like submanifold satisfying (1.3) in $\mathbb{R}_{n}^{2 n}$ with the induced metric $u_{i j} d x_{i} d x_{j}$. Then up to an additive constant the function $u$ is a solution to the Monge-Ampère type equation

$$
\operatorname{det} D^{2} u=\exp \left\{n\left(-u+\frac{1}{2} \sum_{i=1}^{n} x_{i} \frac{\partial u}{\partial x_{i}}\right)\right\} \text {. }
$$

Q. Ding and Y.L. Xin [9] proved that every classical strictly convex entire solutions of the equation (1.4) must be a quadratic polynomial.

Another important examples of Type II singularities is a class of eternal solutions known as translating solitons. From [3], we see that A. Neves and G. Tian gave examples that exclude the existence of nontrivial translating solutions to Lagrangian mean curvature flow. Some interesting translating solitons were found by D. Joyce, Y.I. Lee and M.P. Tsui [1] with oscillation of the Lagrangian angle arbitrarily small. Recently, Martín, Savas-Halilaj and Smoczyk [8] obtained classification results and topological obstructions for the existence of translating solitons of the mean curvature flow in Euclidean space. In this paper we will classify the translating solutions 
of Lagrangian mean curvature flow under certain convexity assumptions on the generating potential as flat Lagrangian planes in pseudo-Euclidean space $\mathbb{R}_{n}^{2 n}$.

A.M. Li and the second author [2] showed that every smooth strictly convex solutions of the Monge-Ampère type equation

$$
\operatorname{det} D^{2} u=\exp \left\{-\sum_{i=1}^{n} d_{i} \frac{\partial u}{\partial x_{i}}-d_{0}\right\}, x \in \mathbb{R}^{n}
$$

must be a quadratic polynomial where $d_{0}, d_{1}, \cdots, d_{n}$ are constants. Here we consider the following more general Monge-Ampère type equation

$$
\operatorname{det} D^{2} u=\exp \left\{\sum_{i=1}^{n}-a_{i} \frac{\partial u}{\partial x_{i}}+\sum_{i=1}^{n} b_{i} x_{i}+c\right\} \quad \text { on } \quad \mathbb{R}^{n},
$$

where $a_{i}, b_{i}$ and $c$ are constants. According to the arguements in [21], the entire solution to (1.5) is a space-like translating soliton to Lagrangian mean curvature flow in pseudo-Euclidean space. As for the authors, it seems that the approach in [18] can't be applied to here for obtaining the rigidity result of the solutions. So we want to search for new ideas to prove the theorems similar to Jörgens [15], Calabi [12], and Pogorelov [4].

Let $A$ be an $n \times n$ real matrix and define

$$
\Lambda=\left\{l \mid A^{l}=I, A^{l-1} \neq I, l \in Z^{+}\right\}, \quad l_{A}=\min _{l \in \Lambda} l .
$$

Denote $a=\left(a_{1}, a_{2}, \cdots, a_{n}\right), b=\left(b_{1}, b_{2}, \cdots, b_{n}\right)$ and $\langle a, b\rangle$ is the inner product of two vectors in $\mathbb{R}^{n}$. As an application of Proposition [1.2, we can prove that

Theorem 1.1. Let $u$ be a smooth strictly convex solution of (1.5) $(n \geq 2)$ where $|a| \neq 0,|b| \neq 0$. Suppose that there exists an orthogonal matrix $A$ such that $l_{A} \geq 3$ and $u(A x)=u(x)$ for each $x \in \mathbb{R}^{n}$. If the smallest eigenvalue $\mu(x)$ of $D^{2} u$ satisfies

$$
\liminf _{x \rightarrow \infty}|x| \mu(x)>\frac{n-1}{|a| \cos \frac{\pi}{l_{A}}},
$$

then $u(x)$ must be a quadratic polynomial.

By the methods in the proof of Theorem 1.1, we formulate the above result in a more general form when the dimension $n=1$ :

Corollary 1.1. Suppose that $u=u(t)$ satisfies

$$
u^{\prime \prime}=\exp \left(-a_{0} u^{\prime}+b_{0} t+c\right) \quad \text { on } \quad \mathbb{R},
$$

where $a_{0} b_{0}>0$ and there exists $t_{0}$ such that $u\left(t-t_{0}\right)=u\left(t_{0}-t\right)$ for each $t \in \mathbb{R}$. Then

$$
u=\frac{b_{0}}{2 a_{0}}\left(t-t_{0}\right)^{2}+\min _{\mathbb{R}} u .
$$

If the potential function $u$ has more symmetry, it is easy to get

Theorem 1.2. Let $u$ be a smooth strictly convex radially symmetric solution of (1.5), then $u(x)$ must be a quadratic polynomial. 
We outline our proof as follows. In section 2 , we provide preliminary results which will be used in the proof of Theorem 1.1. The techniques used in this section are reflective of those in [20], but the corresponding prior estimates to the solutions in the current scenario need modification because the structure of (1.5) is unlike the self-shrinking equation (1.4). In section 3 , we give the proofs of the main results.

\section{Preliminaries}

Straightforward computation gives the relations of (1.1) and (1.5).

Lemma 2.1. If $u$ is a smooth strictly convex solution of the PDE (1.5) and define $\tilde{u}(x, t)=u(x-a t)+\left(\langle b, x\rangle-\frac{1}{2}\langle b, a\rangle t+c\right) t$. Then $\tilde{u}(x, t)$ satisfies the logarithmic Monge-Ampère flow

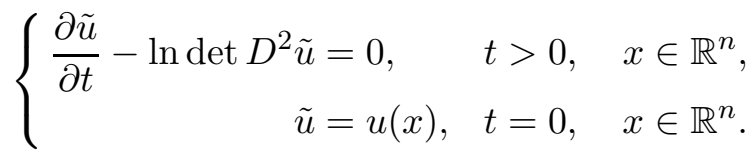

An important consequence of the decay estimates (1.2) is the following result.

Lemma 2.2. If $u$ is a smooth strictly convex solution of the PDE (1.5) and satisfies condition (S). Then u(x) must be a quadratic polynomial.

Proof. By Lemma 2.1 and Proposition 1.2, we have

$$
\sup _{x \in \mathbb{R}^{n}}\left|D^{3} \tilde{u}(x, t)\right|^{2} \leq \frac{C}{t}, \quad \forall t \geq \varepsilon_{0} .
$$

That is

$$
\sup _{x \in \mathbb{R}^{n}}\left|D^{3} u(x-a t)\right|^{2} \leq \frac{C}{t}, \quad \forall t \geq \varepsilon_{0}
$$

Therefore

$$
\sup _{x \in \mathbb{R}^{n}}\left|D^{3} u(x)\right|^{2} \leq \frac{C}{t}, \quad \forall t \geq \varepsilon_{0} .
$$

Let $t \rightarrow+\infty$ then we obtain $D^{3} u \equiv 0$ and the claim follows.

To obtain the first rigidity theorem we search that which condition can imply condition (S). Denote $B_{R}$ be a ball centered at 0 with radius $R$ in $\mathbb{R}^{n}$.

Lemma 2.3. Let $u: \mathbb{R}^{n} \rightarrow \mathbb{R}$ be a smooth strictly convex solution to (1.5) and $|a| \neq 0$. Suppose that there exists an orthogonal matrix $A$ such that $l_{A} \geq 3$ and $u(A x)=u(x)$ for each $x \in \mathbb{R}^{n}$. If the smallest eigenvalue $\mu(x)$ of $D^{2} u$ satisfies (1.6). Then there exists a positive constant $R_{0}$ such that

$$
D^{2} u(x) \leq C I, \quad x \in \mathbb{R}^{n},
$$

where $C$ is a positive constant depending only on $|a|, l_{A}, \mu(x)$ and $\|u\|_{C^{2}\left(\bar{B}_{R_{0}+1}\right)}$.

Proof. Denote

$$
u_{i}=\frac{\partial u}{\partial x_{i}}, \quad u_{i j}=\frac{\partial^{2} u}{\partial x_{i} \partial x_{j}}, \quad u_{i j k}=\frac{\partial^{3} u}{\partial x_{i} \partial x_{j} \partial x_{k}}, \cdots
$$


and

$$
\left[u^{i j}\right]=\left[u_{i j}\right]^{-1}, \quad L=u^{i j} \frac{\partial^{2}}{\partial x_{i} \partial x_{j}} .
$$

Let $\gamma$ denote a unite vector field. Set

$$
u_{\gamma}=D_{\gamma} u, u_{\gamma \gamma}=D_{\gamma \gamma}^{2} u \text {. }
$$

We will prove that

$$
\sup _{x \in \mathbb{R}^{n}, \gamma \in \mathbb{S}^{n-1}} u_{\gamma \gamma} \leq C .
$$

By (1.6), there are some constant $\lambda>\frac{n-1}{|a| \cos \frac{\pi}{l_{A}}}$ and $R_{0}$, such that

$$
|x| \mu(x) \geq \lambda
$$

for $|x|>R_{0}+1$. One can define a family of smooth functions by

$$
f_{k}(t)=\left\{\begin{array}{lr}
1, & 0 \leq t \leq R_{0}, \\
\varphi & R_{0} \leq t \leq R_{0}+1, \\
-k\left[t^{2}-\left(R_{0}+1\right)^{2}\right]+\frac{3}{4}, & t \geq R_{0}+1,
\end{array}\right.
$$

where $0<k \leq 1$, and $(t, \varphi(t))$ is a smooth curve connecting two points $\left(R_{0}, 1\right)$, $\left(R_{0}+1, \frac{3}{4}\right)$ satisfying $\frac{3}{4} \leq \varphi \leq 1$.

We view $u_{\gamma \gamma}$ as a function on $\mathbb{R}^{n} \times \mathbb{S}^{n-1}$. It is easy to see that $f_{k}(|x|) u_{\gamma \gamma}$ always attains its maximum at

$$
(p, \xi) \in\left\{(x, \gamma) \in \mathbb{R}^{n} \times \mathbb{S}^{n-1} \mid f_{k}(|x|)>0\right\} .
$$

Using the definition of $l_{A}$ we can choose the maximum point $x$, denoted by $p$, such that

$$
\langle x,-a\rangle \geq|a||x| \cos \frac{\pi}{l_{A}} .
$$

By (1.6), we have $u_{\gamma \gamma}>0$. Let

$$
\eta_{k}(x)=f_{k}(|x|), \quad w=\eta_{k}(x) u_{\xi \xi}
$$

Then at $p$,

$$
0 \geq L w=u^{i j}\left(\eta_{k} u_{\xi \xi}\right)_{i j}=u^{i j}\left(\eta_{k}\right)_{i j} u_{\xi \xi}+2 u^{i j}\left(\eta_{k}\right)_{i}\left(u_{\xi \xi}\right)_{j}+\eta_{k} u^{i j}\left(u_{\xi \xi}\right)_{i j} .
$$

We assume that

$$
p \in\left\{x \in \mathbb{R}^{n}|| x \mid>R_{0}+1\right\} .
$$

By a rotation, we can assume that $D^{2} u$ is diagonal at $p$ with $\xi$ as the $x_{1}$ direction. In this case, $u_{\xi \xi}=u_{11}$. Then at $p$, there holds

$$
\left(\eta_{k} u_{11}\right)_{j}=0, \quad j=1,2, \cdots, n .
$$

Hence

$$
\left(u_{11}\right)_{j}=-u_{11} \frac{\left(\eta_{k}\right)_{j}}{\eta_{k}}, \quad\left(\eta_{k}\right)_{j}=-\eta_{k} \frac{\left(u_{11}\right)_{j}}{u_{11}}, \quad j=1,2, \cdots, n .
$$


Clearly, by (2.4),

$$
\begin{aligned}
2 u^{i j}\left(\eta_{k}\right)_{i}\left(u_{11}\right)_{j} & =u^{11}\left(\eta_{k}\right)_{1} u_{111}+u^{11}\left(\eta_{k}\right)_{1} u_{111}+2 \sum_{i \neq 1} \frac{\left(\eta_{k}\right)_{i} u_{11 i}}{u_{i i}} \\
& =-u^{11} \frac{\left(\eta_{k}\right)_{1}\left(\eta_{k}\right)_{1}}{\eta_{k}} u_{11}-u^{11} \eta_{k} \frac{u_{111}^{2}}{u_{11}}-2 \sum_{i \neq 1} \eta_{k} \frac{u_{11 i}^{2}}{u_{i i} u_{11}} .
\end{aligned}
$$

Differentiating the equation (1.5), we have

$$
\begin{gathered}
u^{i j} u_{i j 1}=-a_{i} u_{i 1}+b_{1}, \\
u^{i j} u_{11 i j}=\sum_{i, j=1}^{n} \frac{u_{i j 1}^{2}}{u_{i i} u_{j j}}-a_{i} u_{i 11} .
\end{gathered}
$$

Substituting (2.5), (2.6) into (2.3) and using

$$
\left(\eta_{k}\right)_{i}=-2 k x_{i}, \quad\left(\eta_{k}\right)_{i j}=-2 k \delta_{i j}
$$

we obtain, at $p$,

$$
\begin{aligned}
0 \geq & -2 k \sum_{i=1}^{n} u^{i i} u_{11}-\frac{\left(\eta_{k}\right)_{1}^{2}}{\eta_{k}}-\eta_{k} \frac{u_{111}^{2}}{u_{11}^{2}}-2 \eta_{k} \sum_{i \neq 1} \frac{u_{11 i}^{2}}{u_{i i} u_{11}} \\
& +\eta_{k} \sum_{i, j=1}^{n} \frac{u_{i j 1}^{2}}{u_{i i} u_{j j}}-\eta_{k} a_{i} u_{i 11} .
\end{aligned}
$$

Note that

$$
\eta_{k} \sum_{i, j=1}^{n} \frac{u_{i j 1}^{2}}{u_{i i} u_{j j}} \geq \eta_{k} \frac{u_{111}^{2}}{u_{11}^{2}}+2 \eta_{k} \sum_{i \neq 1} \frac{u_{11 i}^{2}}{u_{i i} u_{11}} .
$$

Combining the above two inequalities, we get

$$
0 \geq-2 k \sum_{i=1}^{n} u^{i i} u_{11}-\frac{\left(\eta_{k}\right)_{1}^{2}}{\eta_{k}}-\eta_{k} a_{i} u_{i 11} .
$$

In view of (2.4),

$$
\eta_{k} u_{i 11}=-u_{11}\left(\eta_{k}\right)_{i}=2 k x_{i} u_{11}
$$

Then at $p$,

$$
\frac{\left(\eta_{k}\right)_{1}^{2}}{\eta_{k}} \geq-2 k \sum_{i=1}^{n} u^{i i} u_{11}-2 k a_{i} x_{i} u_{11} .
$$

Using (2.2) and $u_{i i} \geq \frac{\lambda}{|x|}$ for $i \geq 2$, it follows from the above arguments that

$$
\frac{4 k^{2} x_{1}^{2}}{\eta_{k}} \geq-2 k-\frac{2 k(n-1)}{\lambda}|x| u_{11}+2 k|a||x| u_{11} \cos \frac{\pi}{l_{A}}
$$


Noting $\lambda>\frac{n-1}{|a| \cos \frac{\pi}{l_{A}}}$, at $p$, we have

$$
\frac{1}{\left(|a| \cos \frac{\pi}{l_{A}}-\frac{n-1}{\lambda}\right)|x|}\left(\eta_{k}+2 k x_{1}^{2}\right) \geq \eta_{k} u_{11} .
$$

Thus if $p \in\left\{x \in \mathbb{R}^{n}|| x \mid>R_{0}+1\right\}$, then there holds

$$
\max _{x \in \mathbb{R}^{n}, \gamma \in \mathbb{S}^{n-1}} \eta_{k} u_{\gamma \gamma} \leq \frac{\frac{3}{4}+2 k\left(R_{0}+1\right)^{2}}{\left(|a| \cos \frac{\pi}{l_{A}}-\frac{n-1}{\lambda}\right)\left(R_{0}+1\right)} .
$$

And if $p \in\left\{x \in \mathbb{R}^{n}|| x \mid \leq R_{0}+1\right\}$, then

$$
\max _{x \in \mathbb{R}^{n}, \gamma \in \mathbb{S}^{n-1}} \eta_{k} u_{\gamma \gamma} \leq\|u\|_{C^{2}\left(\bar{B}_{R_{0}+1}\right)} .
$$

From (2.7) and (2.8), by $k \leq 1$ we obtain

$$
\max _{x \in \mathbb{R}^{n}, \gamma \in \mathbb{S}^{n-1}} \eta_{k} u_{\gamma \gamma} \leq \frac{11\left(R_{0}+1\right)}{4\left(|a| \cos \frac{\pi}{l_{A}}-\frac{n-1}{\lambda}\right)}+\|u\|_{C^{2}\left(\bar{B}_{R_{0}+1}\right)} .
$$

For any fixed $x \in \mathbb{R}^{n}$ and $\gamma \in \mathbb{S}^{n-1}$, let $k$ converges to 0 , then

$$
\frac{3}{4} u_{\gamma \gamma} \leq \frac{11\left(R_{0}+1\right)}{4\left(|a| \cos \frac{\pi}{l_{A}}-\frac{n-1}{\lambda}\right)}+\|u\|_{C^{2}\left(\bar{B}_{R_{0}+1}\right)}
$$

So we obtain

$$
u_{\gamma \gamma} \leq \frac{11\left(R_{0}+1\right)}{3\left(|a| \cos \frac{\pi}{l_{A}}-\frac{n-1}{\lambda}\right)}+\frac{4}{3}\|u\|_{C^{2}\left(\bar{B}_{R_{0}+1}\right)}
$$

and inequality (2.1) is proved.

\section{PROOF OF THE MAIN RESULTS}

Proof of Theorem 1.1;

Introduce Legendre transformation of $u$ :

$$
\tilde{x}_{i}=\frac{\partial u}{\partial x_{i}}, i=1,2, \cdots, n, u^{*}\left(\tilde{x}_{1}, \cdots, \tilde{x}_{n}\right):=\sum_{i=1}^{n} x_{i} \frac{\partial u}{\partial x_{i}}-u(x), x \in R^{n} .
$$

In terms of $\tilde{x}_{1}, \cdots, \tilde{x}_{n}, u^{*}\left(\tilde{x}_{1}, \cdots, \tilde{x}_{n}\right)$, one can easily check that

$$
\left(\frac{\partial^{2} u^{*}}{\partial \tilde{x}_{i} \partial \tilde{x}_{j}}\right)=\left(\frac{\partial^{2} u}{\partial x_{i} \partial x_{j}}\right)^{-1}
$$

Thus, in view of (2.1),

$$
D^{2} u^{*} \geq \frac{1}{C} I .
$$


By the PDE (1.5) we obtain

$$
\operatorname{det} D^{2} u^{*}=\exp \left\{a_{i} \tilde{x}_{i}-b_{i} u_{i}^{*}-c\right\} .
$$

Since $u(A x)=u(x)$ one can verify that $u^{*}(A \tilde{x})=u^{*}(\tilde{x})$ for each $\tilde{x} \in \mathbb{R}^{n}$. Using Lemma 2.3, we have

$$
D^{2} u^{*} \leq C I
$$

So

$$
\frac{1}{C} I \leq D^{2} u \leq C I
$$

An application of Lemma 2.2 yields the desired result.

Proof of Corollary 1.1;

Without loss of generality, we assume that $t_{0}=0$. The equation (1.7) shows that $u$ must be strictly convex. For $n=1$, similar to the proof of Lemma 2.3, the inequality (2.2) can be replaced by

$$
\left\langle t,-a_{0}\right\rangle \geq\left|a_{0}\right||t| .
$$

Here we use the symmetry condition. Following the procedure in the proof of Theorem 1.1, we obtain estimates (2.1) for $n=1$ and then we arrive at the conclusion of Corollary 1.1 ,

Proof of Theorem 1.2; Now we assume that $u$ is radially symmetric function, then

$$
\Psi=\ln \operatorname{det}\left(u_{i j}\right)=\ln u_{r r}+(n-1)\left(\ln u_{r}-\ln r\right)
$$

is also radially symmetric and depends only on $|x|$. Similar to the arguments in [1], it follows that $\ln \operatorname{det}\left(u_{i j}\right)$ must then attain either alocal maximum or a local minimum over any open ball $B$ in $\mathbb{R}^{n}$. From (1.5), we have

$$
u^{i j} \Psi_{i j}-\langle a, D \Psi\rangle=0 \text {. }
$$

Applying the strong maximum principle to (3.1), we see that $\Psi$ is constant in $B$, and hence in $\mathbb{R}^{n}$. From the classical Jorgens-Calabi-Pogorelov theorem, we complete the proof of theorem.

Acknowledgment: The first author was supported by NNSF of China (Grant No.11261008) and NNSF of Guangxi (Grant No.2012GXNSFBA053009) and was very grateful to Institute of Differential Geometry at Leibniz University Hannover for the kind hospitality. The second author was partially supported by NSFC (Grant No.11101129).

\section{REFERENCES}

[1] A. Chau, J.Y. Chen, Y. Yuan: Rigidity of Entire self-shrinking solutions to curvature flows, J. Reine Angew. Math. 664(2012), 229-239.

[2] A.M. Li, R.W. Xu: A rigidity theorem for an affine Kähler-Ricci flat graph, Results Math. 56(2009), 1-24.

[3] A. Neves, G. Tian:Translating solutions to Lagrangian mean curvature flow, Trans. Amer. Math. Soc. 365(2013), 5655-5680.

[4] A.V. Pogorelov: On the improper convex affine hyperspheres, Geom. Dedicata. 1(1972), 33-46.

[5] A.M. Li, R.W. Xu, U. Simon, F. Jia: Affine Bernstein problems and Monge-Ampère equations, World Scientific Publishing Co. Singapore, 2010. 
[6] Q.M. Cheng: Estimates for eigenvalues of L operator on self-shrinkers, arXiv:1112.5938, 2011.

[7] Q.M. Cheng: Complete self-shrinkers of the mean curvature flow, arXiv:1202.1053, 2012.

[8] F. Martín, A. Savas-Halilaj, K. Smoczyk: On the topology of translating solitons of the mean curvature flow, arXiv:1404.6703v1, 2014.

[9] Q. Ding, Y.L. Xin: The rigidity theorems of Lagrangian self shrinkers, J. Reine Angew. Math. 692(2014), 109-123.

[10] Q. Ding, Z.Z. Wang: On the self-shrinking systems in arbitrary codimension spaces, arXiv:1012.0429, 2010.

[11] D. Joyce, Y.I. Lee and M.P. Tsui: Self-similar solutions and translating solitons for Lagrangian mean curvature flow, J. Differential Geom. 84(2010), 127-161.

[12] E. Calabi: Improper affine hypersurfaces of convex type and a generalization of a theorem by K. Jörgens, Michigan Math.J. 5(1958), 105-126.

[13] H.Z. Li: Lower volume growth estimates for self-shrinkers of mean curvature flow, arXiv:1112.0828, 2011.

[14] I. Castro: The Clifford torus as a self-shrinker for the Lagrangian mean curvature flow, arXiv:1202.2555, 2012.

[15] K. Jörgens: ber die Lösungen der Differentialgleichung $r t-s^{2}=1$, Math. Ann. 127(1954), 130134.

[16] K. Smoczyk: Self-shrinkers of the mean curvature flow in arbitrary codimension, Int. Math. Res. Not.IMRN 48(2005), 2983-3004.

[17] K.S. Tso: On a real Monge-Ampere functional. Invent. Math. 101(1990), 425-448.

[18] L. Caffarelli, Y.Y. Li: An extension to a theorem of Jörgens, Calabi, and Pogorelov, Comm. Pure Appl. Math. 56(2003), 549-583.

[19] R.L. Huang: Lagrangian mean curvature flow in pseudo-Euclidean Space, Chin. Ann. Math. Ser.B 32(2011), 187-200.

[20] R.L. Huang, Z.Z. Wang: On the entire self-shrinking solutions to Lagrangian mean curvature flow, Calc. Var. Partial Differential Equations. 41 (2011), 321-339.

[21] R.W. Xu, R.L. Huang: On the rigidity theorems for Lagrangian translating solitons in pseudoEuclidean space I, Acta Math. Sin (Engl. ser). 29(7)(2013), 1369-1380.

[22] R. Schoen, L. Simon and S.T. Yau: Curvature estimates for minimal hypersurfaces, Acta Math. 134(1975), 275-288.

School of Mathematics and Statistics, Guangxi Normal University, Guilin, Guangxi 541004, People's Republic of China, E-Mail:Ronglihuangmath@GXnu.edu.CN

College of Mathematics and Information Science, Henan Normal University, XinxIANG, Henan 453007, PeOple's Republic of China, E-MAil:RwXu@Henannu.edu.CN 